\section{LISTENING, LEARNING AND LEADING: DELIVERING TRAINEE-SUGGESTED IMPROVEMENTS TO THE OVERALL FY1 EXPERIENCE USING A NOVEL END OF YEAR SURVEY}

Liam Loftus. FY2 Doctor, Royal Liverpool and Broadgreen University Hospitals NHS Trust

\subsection{6/leader-2019-FMLM.65}

Consensus amongst Foundation Year One (FY1) doctors at the Royal Liverpool University Hospital was that large discrepancies exist in overall FY1 experience between specialties. Discussion with Foundation Programme Directors (FPDs) revealed that no data had ever been collected to assess this.

I therefore conducted a wide-ranging survey that asked both quantitative and qualitative questions focused upon workload and educational opportunities. The survey was distributed at the end of the FY1 year in August 2018, and asked the same set of questions about all three rotations retrospectively. We received 41 responses from a total of 54 FY1s.

I used the data to produce a speciality-specific report for each of our 19 specialities, detailing how their performance compared to other departments. I then worked alongside our FPDs to produce an action plan for each speciality. These highlighted areas that required improvement, with suggestions from FY1s on how to achieve this improvement. FPDs then met with seniors from each specialty to discuss how our action plans could be most effectively implemented.

Resulting improvements include, but are not limited to:

- A number of 'essential actions,' such as rearranging ward round times to ensure FY1s did not routinely work beyond their contracted hours.

- Redistribution of FY1s to areas where workload is more manageable and educational opportunities are greater.

- Introduction of support staff (such as Physician Associates and phlebotomists) in areas where FY1s spend a significant time performing routine tasks.

- A series of 'quick wins,' which included addressing IT issues, and rescheduling departmental teaching to ensure that FY1s could attend.

- An increase in experiences that FY1s found useful, such as supervised clinic and theatre opportunities.

This work demonstrates how a simple intervention, such as collecting feedback and using it to produce action plans, can lead to significant improvements for a large group of people.

\section{MANAGING HEALTHCARE TECHNOLOGY INNOVATION IN THE NHS}

${ }^{1,2}$ Angel Jimenez-Aranda* ${ }^{1,2}$ Liz Pryde. ${ }^{1}$ NIHR Devices for Dignity MedTech Co-operative, UK; ${ }^{2}$ Sheffield Teaching Hospitals NHS Foundation Trust, UK

10.1136/leader-2019-FMLM.66

The NHS Long Term Plan recognises the importance of research and innovation to drive future outcomes improvements and the need to speed up the pipeline for developing and implementing proven and affordable technologies.
However, the innovation pathway in the NHS is complex and requires tools to contribute to the efficient working of the organisation and information sharing.

Devices for Dignity (D4D) is a MedTech Co-operative established by the National Institute for Health Research (NIHR) that has developed an effective model for healthcare technology innovation management.

To satisfy the need to collaboratively manage the innovation activities in $\mathrm{D} 4 \mathrm{D}$, and following the evaluation and rejection of different commercial IT tools, a new web application named InnovationManager was developed in-house.

We conducted an evaluation of InnovationManager using the Computer System Usability Questionnaire (CSUQ). Participants were recruited from the team of NIHR researchers who had been continuously using the system for a minimum period of one year. Ten individuals responded to the invitation to take part in the study and were directed to an online questionnaire, rating each of the 19 items in CSUQ. Responses for each item were summed and averaged to three subscales (Interface Quality, Information Quality, System Usefulness) and an overall scale.

Users found the system very usable and useful, with average scores for each of the subscales and the overall scale above the upper quartile on each 7-point scale. Users highlighted InnovationManager had increased the visibility of their work, facilitated the collaboration with colleagues and partners, reduced duplication of efforts, and supported their daily tasks with shared information.

Results demonstrate the high level of user satisfaction and the suitability of this digital solution to promote collaborative working and accelerate the innovation pathway in the NHS.

\section{MEDICAL PROFESSIONAL STANDARDS AT UNITED LINCOLNSHIRE HOSPITALS}

Jeff Ashby ${ }^{*}$, Neill Hepburn. United Lincolnshire Hospitals NHS Foundation Trust, UK

\subsection{6/leader-2019-FMLM.67}

Background United Lincolnshire Hospitals is a large, rural, multi-site Trust which has been reliant on medical locums. Despite very high-performing doctors, the practice of some fell below the standard. We found that recruitment practices contributed to quality issues.

Objectives/Methods To investigate whether there was an association between quality and the practice of:

1. Appointing locums without specialist registration to Consultant posts.

2. Employing doctors with pre-existing GMC conditions.

The prevalence of doctors with GMC conditions by specialty was established. Incidents by specialty was sourced allowing for comparison.

Information was sourced on doctors who had encountered difficulties that required escalation to the Medical Director.

Results

Specialist Registration (SR)

$18 \%$ of Consultant posts were filled by locums without SR. $42 \%$ of locum Consultants did not hold SR. A correlation was demonstrated between the lack of SR and risk. A moderate positive correlation (0.57) was identified with serious 